Original Research Paper

\title{
Cyclic Prefix Length Determination for Orthogonal Frequency Division Multiplexing System over Different Wireless Channel Models Based on the Maximum Excess Delay Spread
}

\author{
${ }^{1}$ Amar Al-Jzari and ${ }^{2}$ Kostanic Iviva \\ ${ }^{I}$ Department of Electrical Engineering, Technical College, Basrah, Iraq \\ ${ }^{2}$ Department of ECE, Florida Institute of Technology, Melbourne, USA
}

\author{
Article history \\ Received: 14-01-2015 \\ Revised: 22-03-2015 \\ Accepted: 10-04-2015 \\ Corresponding Author: \\ Kostanic Ivica \\ Department of ECE, Florida \\ Institute of Technology, \\ Melbourne, USA \\ Email: kostanic@fit.edu
}

\begin{abstract}
Cyclic Prefix (CP) is one of the key OFDM parameters. It is used to completely eliminate both Inter-Symbol Interference (ISI) and Inter-Carrier Interference (ICI) as long as the CP length is greater than the channel delay spread. By eliminating the ISI and ICI, the CP compensates for the effect of the multi-path dispersion; but it consumes a considerable amount of the scarce spectrum and the power. Contemporary OFDM systems usually use a fixed and large CP length to tolerate worst case channel condition. This technique, however, causes a loss in bandwidth efficiency as well as consumes relatively more transmitter energy. Therefore, there is a need to adopt the CP length based on the channel parameters. This paper investigates the effect of varying the $\mathrm{CP}$ length on the OFDM system over different wireless channel models, where the variable CP length is estimated based on the Maximum Excess Delay Spread (MEDS) of the channel. According to this method, the estimated CP length optimizes the system capacity and improves the overall system performance.
\end{abstract}

Keywords: Cyclic Prefix Length, OFDM System, Power Delay Profiles, Maximum Excess Delay Spread, System Capacity

\section{Introduction}

There is an ever increasing demand to increase the data throughput and to make more efficient use of the available spectrum in wireless data communication systems. The Orthogonal Frequency Division Multiplexing (OFDM) modulation technique is one of the methods used to achieve these goals. Due to its robustness against multi-path fading, the OFDM is becoming a modulation technique of choice for most commercial high-speed broadband wireless communication systems. The OFDM is a special form of multi-carrier modulation schemes that is capable of overcoming the frequency selectivity of the radio channels and providing high data rates without the ISI (Kaur and Bharti, 2012; Marchetti et al., 2009; Prasad, 2004). However, in multipath fading channels, the time variation of a fading channel over an OFDM symbol period destroys the orthogonality between the sub- channel and leads to the ICI ( $\mathrm{Li}$ and Kavehrad, 1999; Zhang and Liu, 2006).

A circular extension known as the $\mathrm{CP}$ is added at the head of OFDM symbols in order to eliminate both ISI and ICI. To achieve this goal, the CP length must be greater than the delay spread of the multipath channel (Shah et al., 2010). However the introduction of the CP reduces the bandwidth efficiency and decreases the data rate (capacity of the system) because it conveys no information. It also disperses the transmitter energy (the amount of consumed power relies on how large $\mathrm{CP}$ length), where the Signal-to-Noise (SNR) lost due to the $\mathrm{CP}$ introduction indicates the loss of transmission energy. Because of the loss of SNR and the bandwidth efficiency, the CP needs to be chosen optimally. Typically, it is chosen based on the multipath channel duration in a given operating environment (Ness et al., 2002; Payaswini and Manjaiah, 2013). 
The CP is always appended to the head of the OFDM symbols. This result in loss of spectral efficiency of the system. For example, in IEEE $802.11 \mathrm{a} / \mathrm{g}$ standard about $25 \%$ of the useful signal is utilized for the CP $[9,10]$. Therefore, it is essential to understand the $\mathrm{CP}$ adaptation scheme. The conventional OFDM uses a large and fixed $\mathrm{CP}$ length to tolerate worst case channel condition irrespective of current channel state. This technique, however will lead to loss in bandwidth efficiency as well as waste of the transmit energy proportional to the $\mathrm{CP}$ length. If the receiver is designed based on indoor delay spread, the mobile terminal will undergo severe ISI when it moves to outdoor. Conversely, if the receiver is designed based on outdoor delay spread, some of the guard band will be unnecessary, which wastes the spectrum and increases battery power consumption. Therefore would be beneficial to change the CP length based on the channel parameters (Hassan et al., 2011). In (Das et al., 2005; Ghosh, 2011) OFDM uses a CP which is selected to be several times more than the RMS delay spread of the operating environment. This CP selection mandates devices which experience smaller RMS delay spread to use an excessively longer $\mathrm{CP}$, in this situation the scarce network resource is misused and the limited battery power is wasted from the device perspective. This fact motivates the research into ways for making the CP length shorter and even adaptive, which can introduce a significant enhancement in spectral efficiency. In 2004, Zhang proposed a method to adopt the CP length based on the variation of the channel delay spread. His method suggested selection of the CP length to be twice the channel RMS delay spread (Zhang and Lai, 2004). In 2005, Chiwoo, Youngbin, Panyuh, Hyeonwoo and Jaeweon proposed another method to adopt the CP length via adjusting the sampling rate. The drawback of this method is that it requires a wide OFDM spectrum; since the CP is extended by sampling up, the occupied bandwidth will also increase (Lim et al., 2005). In previous work, the Power Delay Profile (PDP) of the channels is fitted into a Negative Exponentially Decaying Profile (NEDP). Based on the NEDP a mathematical formula is derived to estimate the variable CP length for the different channel models.

The evaluation of the $\mathrm{CP}$ length requirements for different mobile environments is the problem addressed in this research. In this study, the variable CP length is adopted based on the last tap of the Power Delay Profile (PDP) of the channel where the PDP is not considered as the NEDP. This means that the CP length is dependent on the MEDS of the channel.

\section{System Model Description}

Figure 1 shows a basic model of an OFDM system. First the input binary data are generated and converted to the data symbols by using different modulation schemes.
Once the sequence of binary values is mapped to a data symbol form, the next step is to generate an OFDM waveform by converting the serial data symbol sequence into a number of shorter parallel symbol sequences. These modulated signals are processed by the IFFT block. Then the $\mathrm{CP}$ is added to the signals. The CP extends the OFDM symbol by copying the last samples of the OFDM symbol and appending it in front of the transmitted OFDM symbol. The reason for that is to preserve the orthogonally between the sub-carriers. Also, the $\mathrm{CP}$ allows the receiver to integrate over an integer number of sinusoid cycles for each of the multipaths when it performs OFDM demodulation with the FFT. After adding the CP, the signals are converted to serial form and transmitted through the channel (Nolan, 2005). At the receiver side, a reverse process is performed. The serial data are received and converted to the parallel form. Then cyclic prefix is removed. After removal of cyclic prefix, Fast Fourier Transform is performed. Then, the signals are demodulated using different modulation schemes to get the original binary data (Nolan, 2005).

Figure 2 shows the proposed OFDM system model. According to this model, at the transmitter end the signal is modulated and subsequently the CP controller attaches the CP length adaptively to mitigate both ISI and ICI introduced due to the channel. At the receiver end, the $\mathrm{CP}$ controller detaches the $\mathrm{CP}$ length before demodulator in order to recover the transmitted signal.

\section{Analysis: SNR and Data Rate Loss beyond the CP Introduction}

To evaluate the effect of the CP length on the OFDM system performance, it is simulated based on the parameters in Table 1 and using different channels PDP such as ITU-A, ITU-B, EITU and 3GPP channel models. This section also indicates how to calculate the loss in data rate and SNR due to the CP insertion based on the OFDM system.

\section{CP Length Calculation}

The OFDM symbol consists of different sub-channels that carry data subcarriers, pilot subcarriers that are used for channel estimation and synchronization purposes, DC subcarrier and guard subcarriers that are used to provide high inter-channel interference margin. To keep the subcarrier spacing fixed at $10.9375 \mathrm{KH}_{\mathrm{Z}}$ across different channel bandwidth, scalability feature of OFDM chooses 1024 FFT length with $10 \mathrm{MH}_{\mathrm{Z}}$ occupied bandwidth. Thus, the useful symbol length $\left(T_{u}\right)$ is inverse of the subcarrier spacing $\Delta f$. Then, the CP length is $\left(T_{g}=\right.$ $\left.G^{*} T_{u}\right)$, where $G$ is $\left(T_{g} / T_{u}\right)$ ratio. The choice of $\mathrm{G}$ is made according to channel parameters. The total OFDM symbol length consists of the useful symbol length and the CP length $\left(T_{O F D M}=T_{g}+T_{u}\right)$. 


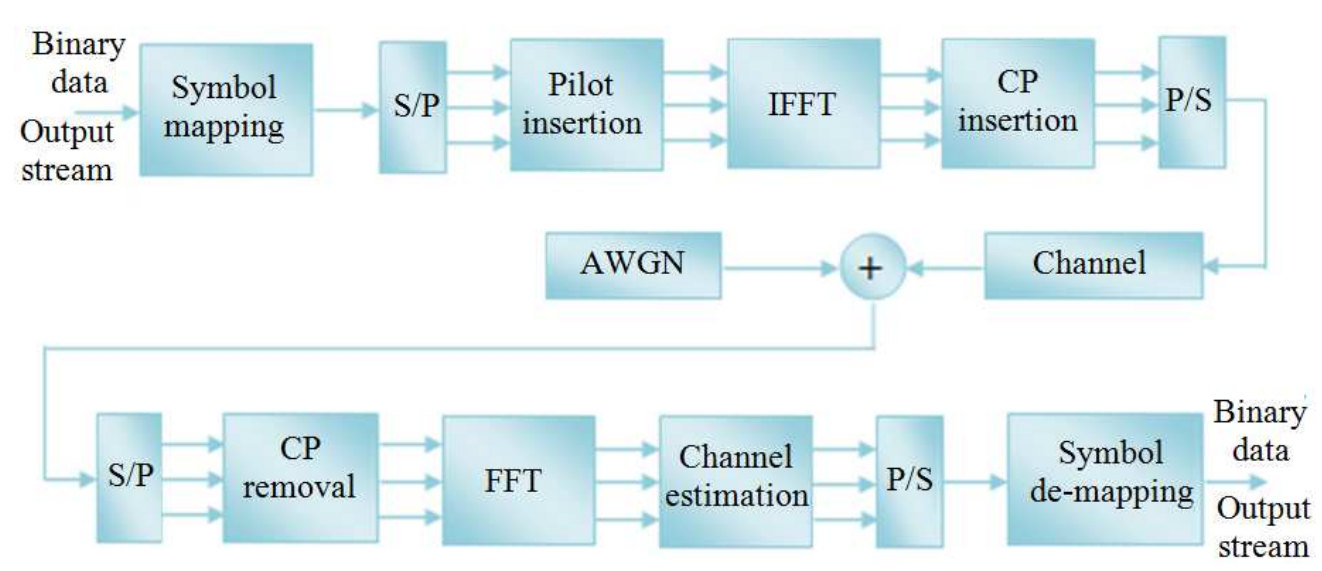

Fig. 1. Block diagram of OFDM system

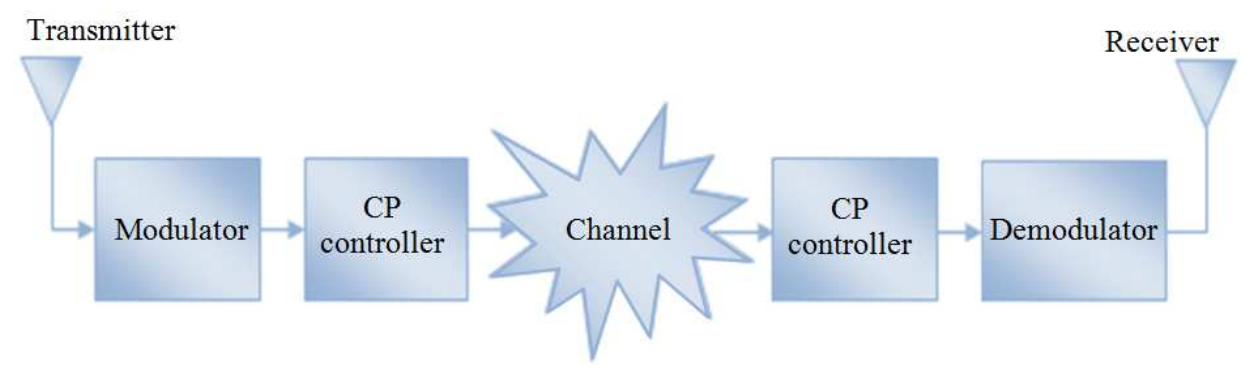

Fig. 2. Block diagram of proposed OFDM system

Table 1. OFDM simulator primitive parameters

\begin{tabular}{ll}
\hline System parameter & Value \\
\hline Bandwidth & $10 \mathrm{MHz}$ \\
Number of data sub-carrier $\left(N_{\text {data }}\right)$ & 560 \\
Number of pilot sub-carrier & 280 \\
Guard sub-carrier & 184 \\
FFT size & 1024 \\
Sampling frequency & $11.2 \mathrm{MHz}$ \\
Subcarrier spacing $(\Delta f)$ & $10.9375 \mathrm{KHz}$ \\
Useful symbol time $(T u=1 / \Delta f)$ & $91.43 \mu \mathrm{s}$ \\
Ratio of CP time to useful time $(\mathrm{G})$ & Variable \\
Cyclic prefix $\left(T_{g}\right)$ & $T_{g}=\mathrm{G}^{*} T u$ \\
Modulation scheme & QPSK \\
\hline
\end{tabular}

\section{Data Rate}

The main goal of the communication system is to achieve high data rate with minimum BER. The maximum transmission data rate can be written as:

$$
R=\frac{N_{\text {data }} \times b}{T_{g}+T_{u}}
$$

where, $b$ is the number of bits per symbol based on the modulation scheme used. It is clear that, by changing the CP length from $3 \%$ of the symbol length to $25 \%$ reduces the amount of data transmitted rate significantly.

\section{SNR Loss}

As the CP length is increased to overcome ISI and ICI, it reduces the overall power efficiency. The SNR lost at the transmitter side can be given by:

$$
S N R_{\text {loss }}=-10 \log _{10}\left(1-\frac{T g}{T o f d m}\right)
$$

At the receiver side, the receiver energy does not change as result of removing the $\mathrm{CP}$. Minimizing power losses is so important because the mobile terminal need to be run on battery. Since the OFDM useful length is fixed the minimization of the power loss can be done by changing the CP length.

\section{Wireless Standard Channel Models}

Several wireless channel models are used to simulate the radio wave propagation. These channels are currently utilized in industry where each model is convenient for a specific type of environment. Uses of standardized models allow researchers to provide 'apple to apple' comparisons of the obtained results. The considered channel models that are used for the performance evaluation tasks in this paper work are: 


\section{ITU Channel Models}

The ITU channel models consist of two types of the channels. Table 1 and 2 show the PDPs and the characteristic of these channels. Theses Tables indicate the relative delay, the average power for the taps of the multi-path channel and the RMS delay spread based on the ITU recommendation.

\section{EITU Channel Models}

The Extended ITU channel model for LTE was called Extended Pedestrian-A (EPA), Extended Vehicular-A (EVA) and Extended Typical Urban (ETU). Table 3 represents the PDPs of this channel. This Table shows the channel models parameters such as the excess delay, the average power and the RMS delay spread.

\section{GPP Channel Models}

The 3GPP channel model consists of urban microcell and urban macro-cell channel model. Table 4 shows the tap delays, the average power and the RMS delay spread for these channel models.

\section{Results and Discussion}

\section{OFDM over ITU-A with Different CP Length}

The aim of this section is to evaluate the performance of the OFDM system with different CP lengths over ITU-A channel models. Figure 3 shows the BER performance for different $\mathrm{CP}$ lengths over ITU Indoor-A PDP. It can be seen that when gradually increasing the CP length the BER decreases proportionally. This is due the fact that the BER is affected by the ISI and the power consumption, so the $\mathrm{CP}$ needs to be increased to eliminate the ISI and to require more transmit power to maintain the same BER. From Fig. 3 , the $C P=4(0.34$ $\mu \mathrm{s})$ gives the minimum BER and provides better performance. The achievable data rate the system can get using this length is $12.204 \mathrm{Mbps}$ and the power loss is $0.0161 \mathrm{~dB}$ to accommodate this $\mathrm{CP}$ length, which indicates an improvement over the loss and the data rate obtained subsequently for ITU Pedestrian-A PDP and ITU Vehicular-A PDP. Figure 4 shows the BER performance for different CP lengths over ITU Pedestrian-A PDP. It can be seen that when the CP length increases the BER decreases and the minimum BER occurs at $C P=6(0.46 \mu \mathrm{s})$. It can be observed that when the CP is less than 6 the BER is slightly high, because these lengths are less than the MEDS of this channel, therefore; the $C P=6$ is enough to mitigate the ISI. The achievable data rate the system can get using this length is $12.188 \mathrm{Mbps}$ and the power loss is 0.0218 $\mathrm{dB}$ to accommodate this $\mathrm{CP}$ length. Figure 5 shows the BER performance for different CP lengths over ITU Vehicular-A PDP. It can be seen that when the CP length increases the BER decreases and the minimum BER occurs at $\mathrm{CP}=29(2.81 \mu \mathrm{s})$. This can be used to mitigate the ISI and achieving acceptable data rate. It can also be observed that for the small CP length the system performs poorly because this CP length is not sufficient to eliminate the ISI. The achievable data rate the system can get using this length is $11.884 \mathrm{Mbps}$ and the power loss is $0.131 \mathrm{~dB}$ to accommodate this $\mathrm{CP}$ length. It can be concluded from these figures the BER degrades as the $\mathrm{CP}$ length increases and the optimal $\mathrm{CP}$ required increased as the MEDS of the channel increases. Also, the data rate of the system decreases as the CP length is increased while the SNR lost at the transmitter side decreases as the CP length is decreased.

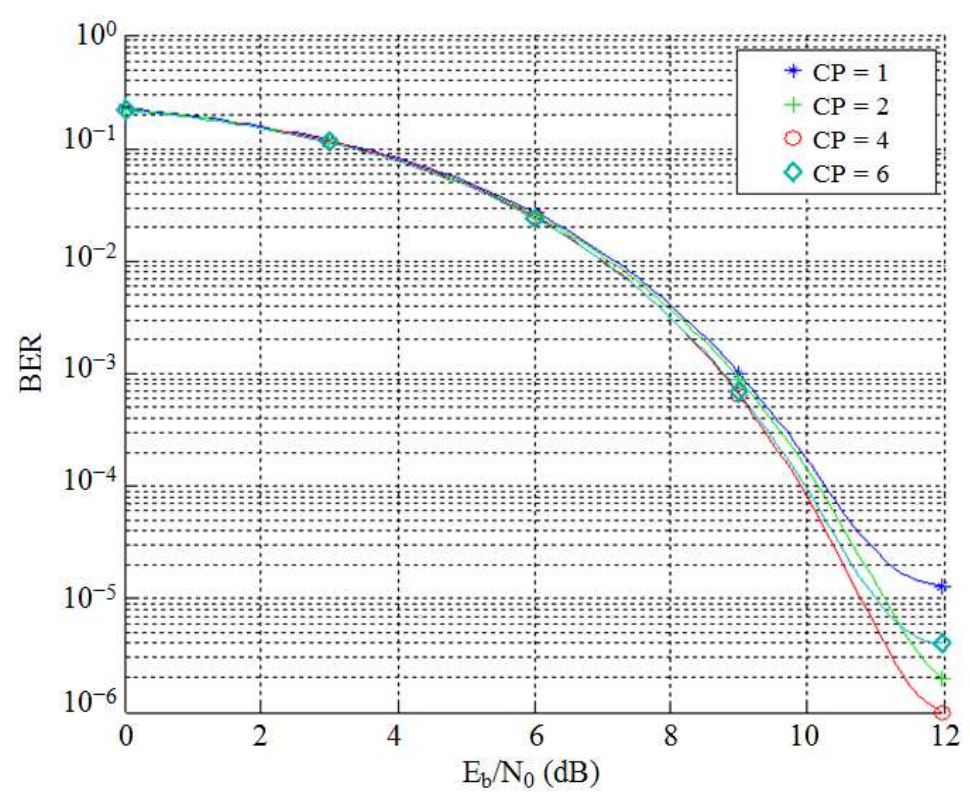

Fig. 3. BER for ITU indoor-A PDP with different CP lengths 


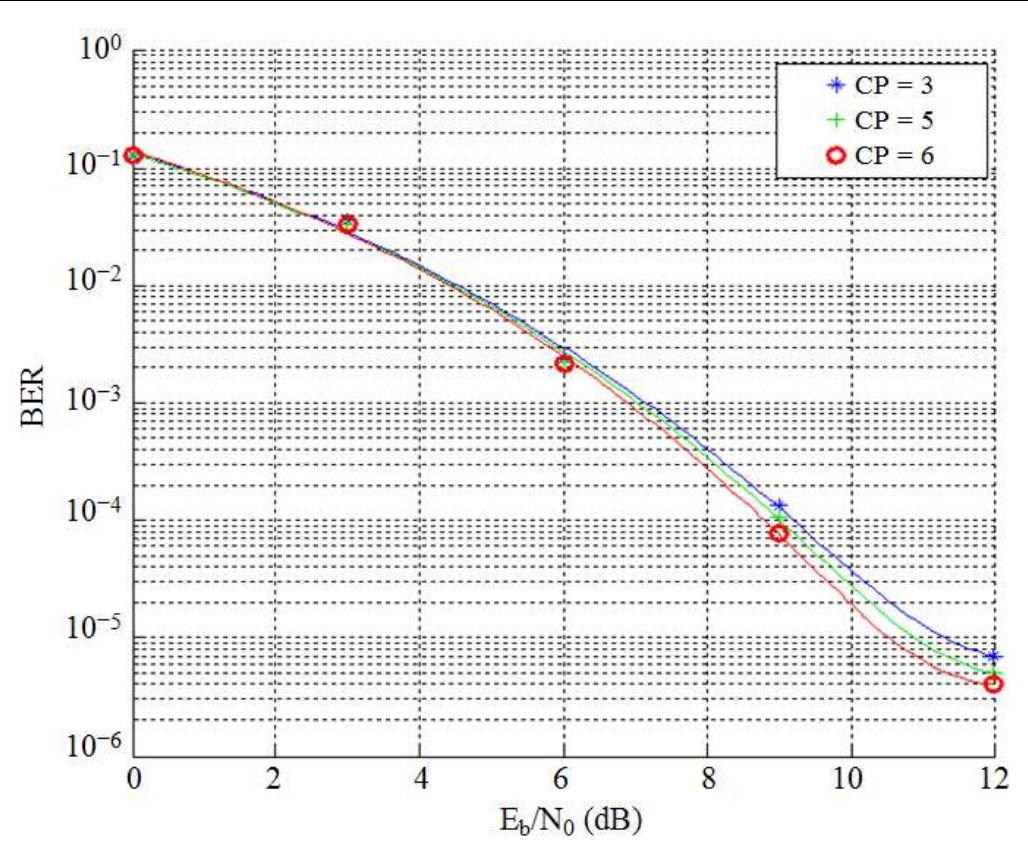

Fig. 4. BER for ITU Pedestrian-A PDP with different CP lengths

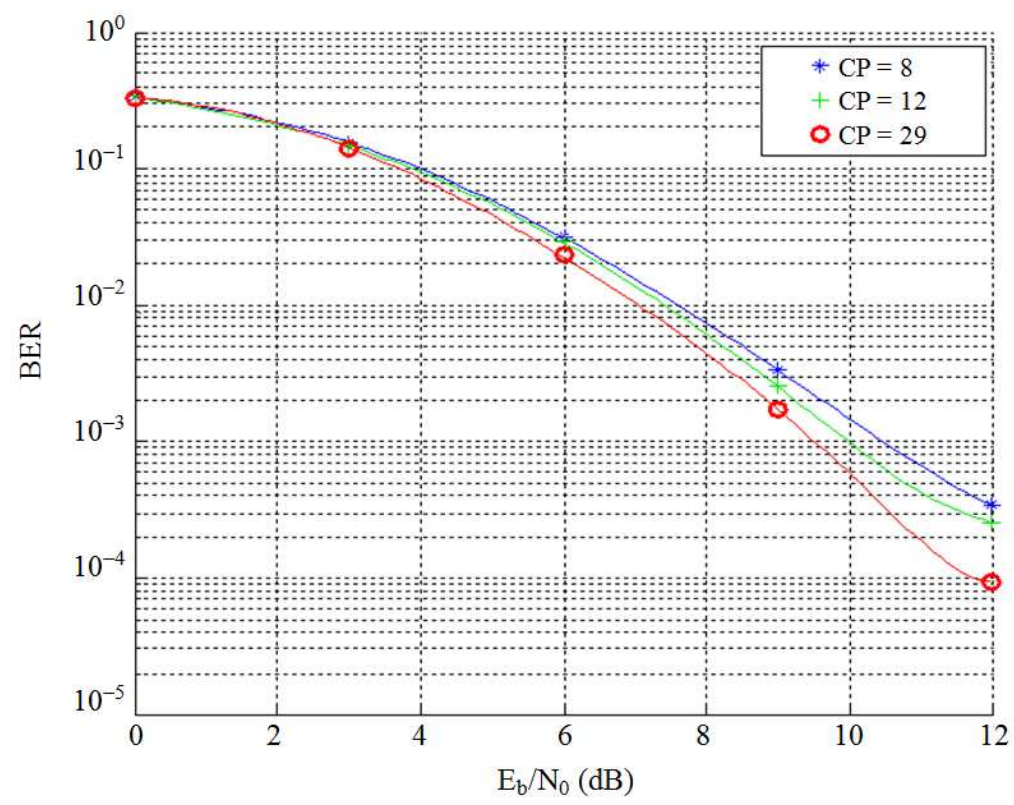

Fig. 5. BER for ITU vehicular-A PDP with different CP lengths

Table 2. Power delay profile of ITU-A channel

\begin{tabular}{|c|c|c|c|c|c|c|}
\hline \multirow[b]{2}{*}{ Tap No. } & \multicolumn{2}{|l|}{ Indoor A } & \multicolumn{2}{|c|}{ Pedestrian A } & \multicolumn{2}{|c|}{ Vehicular A } \\
\hline & Delay (ns) & Power $(\mathrm{dB})$ & Delay (ns) & Power $(\mathrm{dB})$ & Delay (ns) & Power $(\mathrm{dB})$ \\
\hline 1 & 0 & 0 & 0 & 0 & 0 & 0 \\
\hline 2 & 50 & -3.0 & 110 & -9.7 & 310 & -1.0 \\
\hline 3 & 110 & -10.0 & 190 & -19.2 & 710 & -9.0 \\
\hline 4 & 170 & -10.0 & 410 & -22.8 & 1090 & -10.0 \\
\hline 5 & 290 & -26.0 & NA & NA & 1730 & -15.0 \\
\hline \multirow[t]{2}{*}{6} & 310 & -32.0 & NA & NA & 2510 & -20.0 \\
\hline & $\tau_{R M S}=35 n$ & & $\tau_{R M S}=45 n$ & & $\tau_{R M S}=370$ & \\
\hline
\end{tabular}


Amar Al-Jzari and Kostanic Iviva / American Journal of Engineering and Applied Sciences 2015, 8 (1): 82.93 DOI: 10.3844/ajeassp.2015.82.93

Table 3. Power delay profile of ITU-B channel

\begin{tabular}{|c|c|c|c|c|c|c|}
\hline \multirow[b]{2}{*}{ Tap No. } & \multicolumn{2}{|l|}{ Indoor B } & \multicolumn{2}{|c|}{ Pedestrian B } & \multicolumn{2}{|l|}{ Vehicular B } \\
\hline & Delay (ns) & Power $(\mathrm{dB})$ & Delay (ns) & Power $(\mathrm{dB})$ & Delay (ns) & Power $(\mathrm{dB})$ \\
\hline 1 & 0 & 0 & 0 & 0 & 0 & -2.5 \\
\hline 2 & 100 & -3.6 & 200 & -0.9 & 300 & 0 \\
\hline 3 & 200 & -7.2 & 800 & -4.9 & 8900 & -12.8 \\
\hline 4 & 300 & -10.8 & 1200 & -8.0 & 12900 & -10.0 \\
\hline 5 & 500 & -18.0 & 2300 & -7.8 & 17100 & -25.2 \\
\hline \multirow[t]{2}{*}{6} & 700 & -25.2 & 3700 & -23.9 & 20000 & -16.0 \\
\hline & \multicolumn{2}{|c|}{$\tau_{R M S}=100 n s$} & \multicolumn{2}{|c|}{$\tau_{R M S}=750 n s$} & \multicolumn{2}{|c|}{$\tau_{R M S}=4000 n s$} \\
\hline
\end{tabular}

Table 4. Power delay profile of EITU channel

\begin{tabular}{|c|c|c|c|c|c|c|}
\hline \multirow[b]{2}{*}{ Tap No. } & \multicolumn{2}{|l|}{ EPA } & \multicolumn{2}{|l|}{ EVA } & \multicolumn{2}{|l|}{ ETU } \\
\hline & Delay (ns) & Power (dB) & Delay (ns) & Power (dB) & Delay (ns) & Power $(\mathrm{dB})$ \\
\hline 1 & 0 & 0 & 0 & 0 & 0 & -1.0 \\
\hline 2 & 30 & -1.0 & 30 & -1.5 & 50 & -1.0 \\
\hline 3 & 70 & -2.0 & 150 & -1.4 & 120 & -1.0 \\
\hline 4 & 90 & -3.0 & 310 & -3.6 & 200 & 0.0 \\
\hline 5 & 110 & -8.0 & 370 & -0.6 & 230 & 0.0 \\
\hline 6 & 190 & -17.2 & 710 & -9.1 & 500 & 0.0 \\
\hline 7 & 410 & -20.8 & 1090 & -7.0 & 1600 & -3.0 \\
\hline 8 & NA & NA & 1730 & -12 & 2300 & -5.0 \\
\hline \multirow[t]{2}{*}{9} & NA & NA & 2510 & -16.9 & 5000 & -7.0 \\
\hline & \multicolumn{2}{|l|}{$\tau_{R M S}=45 n s$} & \multicolumn{2}{|c|}{$\tau_{R M S}=357 n s$} & \multicolumn{2}{|c|}{$\tau_{R M S}=991 \mathrm{~ns}$} \\
\hline
\end{tabular}

Table 5. Power delay profile of 3GPP channel

\begin{tabular}{|c|c|c|c|c|}
\hline \multirow[b]{2}{*}{ Tap No. } & \multicolumn{2}{|c|}{ Urban macro } & \multicolumn{2}{|c|}{ Urban micro } \\
\hline & Delay (ns) & Power $(\mathrm{dB})$ & Delay (ns) & Power $(\mathrm{dB})$ \\
\hline 1 & 0 & 0 & 0 & 0 \\
\hline 2 & 0.36 & -2.22 & 0.28 & -1.27 \\
\hline 3 & 0.25 & -1.72 & 0.20 & -2.72 \\
\hline 4 & 1.04 & -5.72 & 0.66 & -4.30 \\
\hline 5 & 2.7 & -9.05 & 0.81 & -6.01 \\
\hline \multirow[t]{2}{*}{6} & 4.59 & -12.50 & 0.92 & -8.43 \\
\hline & \multicolumn{2}{|c|}{$\tau_{R M S}=850 \mathrm{~ns}$} & $\tau_{R M S}=295$ & \\
\hline
\end{tabular}

Table 6. OFDM data rate and SNR loss over ITU-A channel model

\begin{tabular}{llll}
\hline Channel type & Optimal CP length & Data rate $(\mathrm{Mbps})$ & Loss $(\mathrm{dB})$ \\
\hline Indoor-A & $4(0.34 \mu \mathrm{s})$ & $12.204 \mathrm{Mbps}$ & $0.0161 \mathrm{~dB}$ \\
Pedestrian-A & $6(0.46 \mu \mathrm{s})$ & $12.188 \mathrm{Mbps}$ & $0.0218 \mathrm{~dB}$ \\
Vehicular-A & $29(2.81 \mu \mathrm{s})$ & $11.884 \mathrm{Mbps}$ & $0.131 \mathrm{~dB}$ \\
\hline
\end{tabular}

The optimal parameters associated with this analysis in order to eliminate both ISI and ICI can be summarized in Table 6 .

\section{OFDM over ITU-B with Different CP Length}

The performance of the OFDM system with different CP lengths is evaluated over ITU-B channel models which consist of ITU Indoor-B PDP, ITU Pedestrian-B PDP and ITU vehicular-B PDP as shown in Table 3. The $\mathrm{CP}$ is also estimated based on the MEDS of the channel. Figure 6 shows the BER performance for different $\mathrm{CP}$ lengths over ITU Indoor-B PDP. It can be seen that when the CP length increases, the BER becomes smaller and the minimum
BER occurs at $C P=8(0.78 \mu \mathrm{s})$. The achievable data rate the system can get using this length is 12.146 Mbps and the loss in the SNR is about $0.0369 \mathrm{~dB}$ to accommodate this CP length. Figure 7 shows the BER performance for different $\mathrm{CP}$ lengths over ITU Pedestrian-B PDP. It can be seen that when the CP length increases, the BER becomes smaller and the minimum BER occurs at $C P=42(4.15 \mu \mathrm{s})$. The achievable data rate the system can get using this length is $11.717 \mathrm{Mbps}$ and the loss in the SNR is about $0.192 \mathrm{~dB}$ to accommodate this $\mathrm{CP}$ length. Figure 8 shows the BER performance for different CP lengths over ITU Vehicular-B PDP. It can be seen that when the $\mathrm{CP}$ length increases, the BER becomes smaller and 
the minimum BER occurs at $C P=224(22.4 \mu \mathrm{s})$. The achievable data rate the system can get using this length is $9.839 \mathrm{Mbps}$ and the loss in the SNR is about $0.951 \mathrm{~dB}$ to accommodate this $\mathrm{CP}$ length. It can be deduced from Fig. 6-8 that ITU-B channels required larger $\mathrm{CP}$ length as compared to ITU-A channel because ITU-B channel has larger delay spread.

The optimal parameters associated with this analysis in order to eliminate both ISI and ICI can be summarized in Table. 7 .

Table 7. OFDM data rate and SNR loss over ITU-B channel model

\begin{tabular}{llll}
\hline Channel type & Optimal CP length & Data rate $(\mathrm{Mbps})$ & Loss $(\mathrm{dB})$ \\
\hline Indoor-B & $8(0.78 \mu \mathrm{s})$ & $12.146 \mathrm{Mbps}$ & $0.0369 \mathrm{~dB}$ \\
Pedestrian-B & $42(4.15 \mu \mathrm{s})$ & $11.717 \mathrm{Mbps}$ & $0.192 \mathrm{~dB}$ \\
Vehicular-B & $224(22.4 \mu \mathrm{s})$ & $9.839 \mathrm{Mbps}$ & $0.951 \mathrm{~dB}$ \\
\hline
\end{tabular}

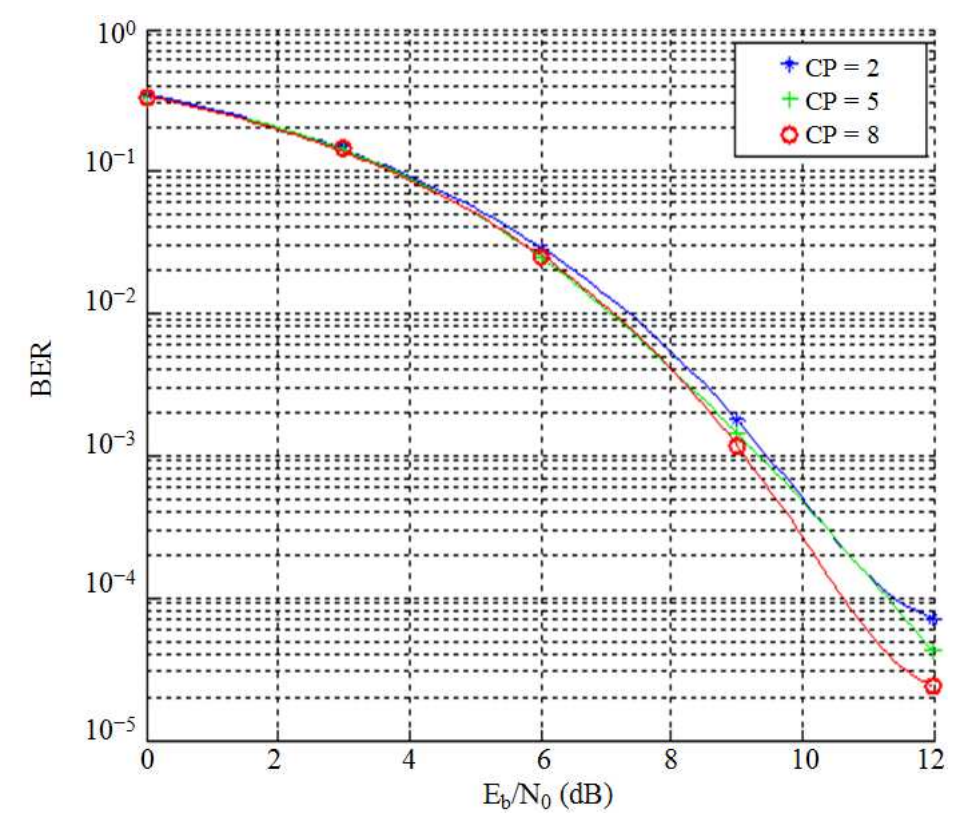

Fig. 6. BER for ITU Indoor-B PDP with different CP lengths

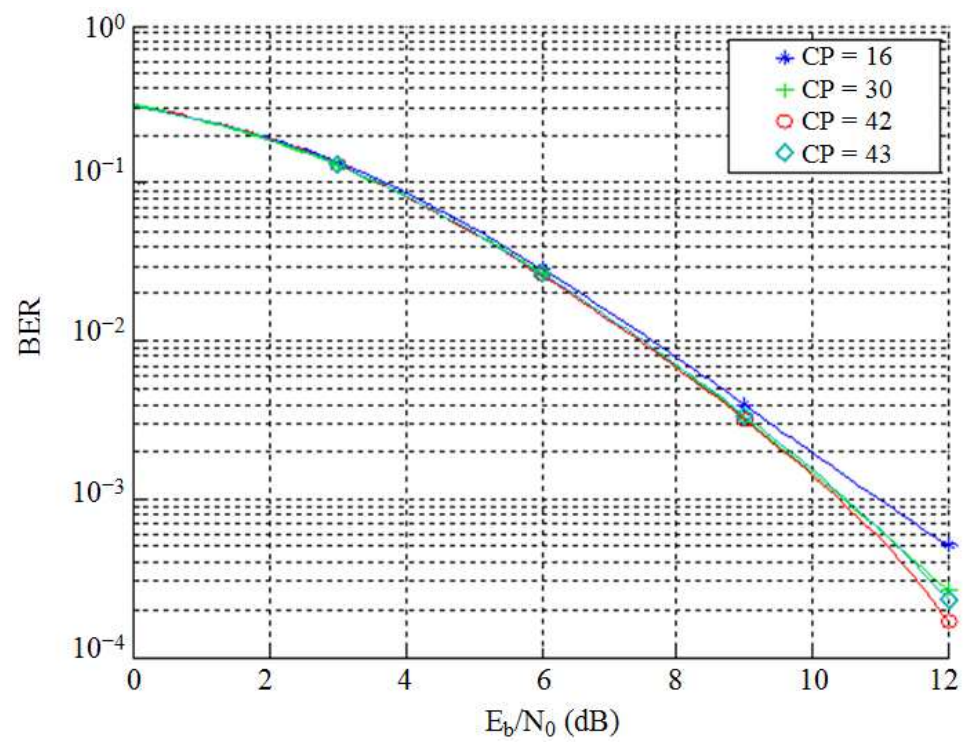

Fig. 7. BER for ITU Pedestrian-B PDP with different CP lengths 


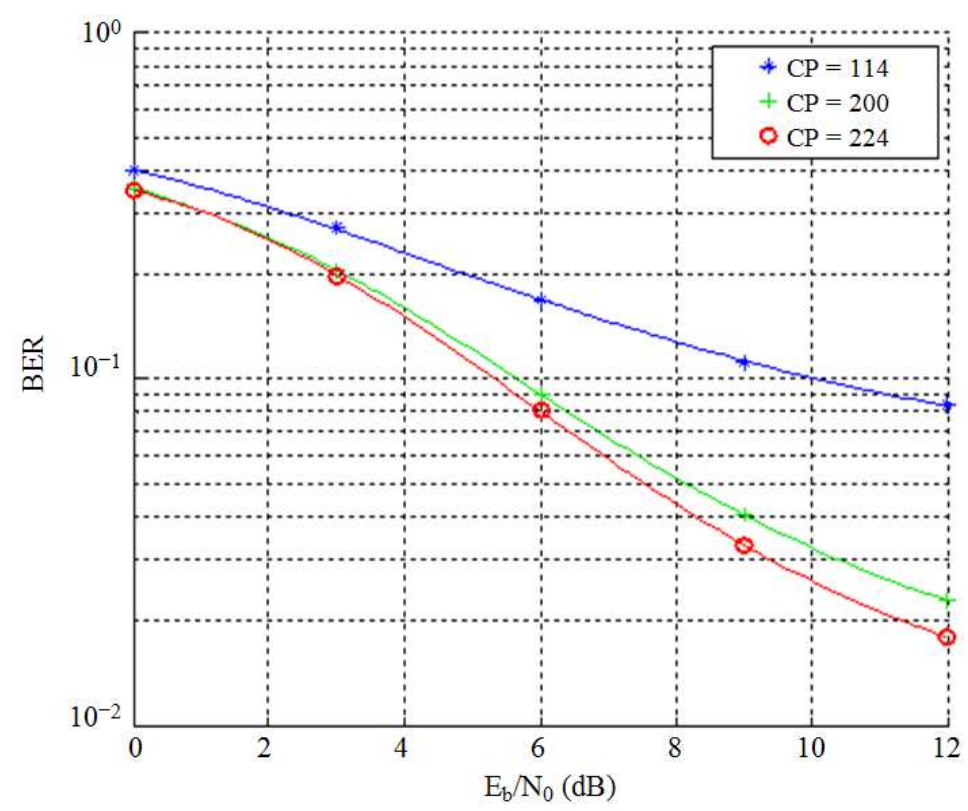

Fig. 8. BER for ITU Vehicular-B PDP with different CP lengths

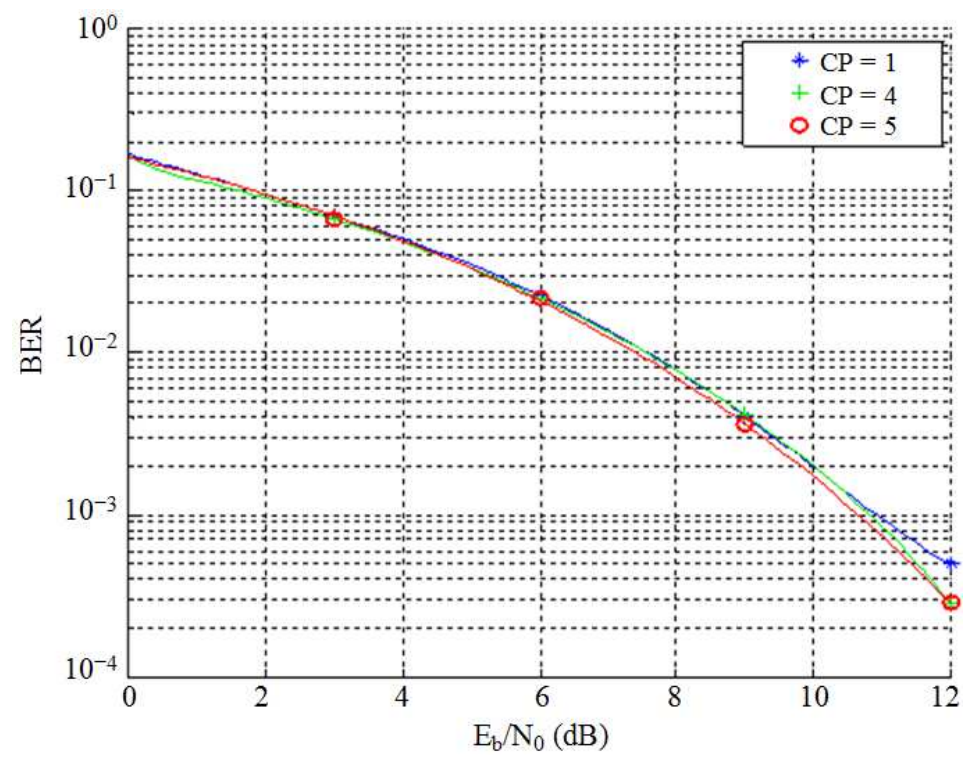

Fig. 9. BER for EPA PDP with different CP lengths

\section{OFDM over EITU with Different CP Length}

The performance of the OFDM system with different $\mathrm{CP}$ lengths is also evaluated over EITU channel models which consist of EPA PDP, EVA PDP and ETU PDP as shown in Table 4. Figure 9 shows the BER performance for different $\mathrm{CP}$ lengths over EPA channel. It may be seen that when the CP length increases, the BER becomes smaller and the minimum BER occurs at $C P=$ $5(0.46 \mu \mathrm{s})$. The achievable data rate the system can get using this length is $12.188 \mathrm{Mbps}$ and the loss in the SNR is $0.0218 \mathrm{~dB}$ to accommodate this $\mathrm{CP}$ length. Figure 10 shows the BER performance for different $\mathrm{CP}$ lengths over EVA channel. It may be seen that when the $\mathrm{CP}$ length increases, the BER becomes smaller and the minimum BER occurs at $C P=29(2.81 \mu \mathrm{s})$. The achievable data rate the system can get using this length is $11.884 \mathrm{Mbps}$ and the loss in the SNR is 0.131 $\mathrm{dB}$ to accommodate this $\mathrm{CP}$ length. Figure 11 shows the BER performance for different $\mathrm{CP}$ lengths over ETU channel. It can be seen that when the CP length increases, the BER becomes smaller and the minimum BER occurs at $C P=56(5.6 \mu \mathrm{s})$. The achievable data rate the system can get using this length is 11.542 
Mbps and the loss in the SNR is $0.258 \mathrm{~dB}$ to accommodate this CP length. It can be seen that ETU PDP has larger CP length as compared to EPA PDP and EVA PDP, because the ETU PDP has larger delay spread, which implies that the transmission over this channel is severely distorted, therefore; the required CP length is relatively large to mitigate this distortion.

The optimal parameters associated with this analysis in order to eliminate both ISI and ICI can be summarized in Table 8.

\section{OFDM over $3 G P P$ with Different CP Length}

The performance of the OFDM system with different CP lengths is also evaluated over 3GPP channel models which consist of urban micro-cell PDP and urban macro-cell PDP as shown in Table 8.
Figure 12 shows the BER performance for different $\mathrm{CP}$ lengths over urban micro-cell PDP. It can be seen that when the CP length increases, the BER becomes smaller and the minimum BER occurs at $\mathrm{CP}=11(1.03$ $\mu \mathrm{s})$. Figure 14 shows the BER performance for different CP lengths over urban macro-cell PDP. It can be seen that when the $\mathrm{CP}$ length increases, the BER becomes smaller and the minimum BER occurs at $\mathrm{CP}=52(5.14 \mu \mathrm{s})$. It can be observed from these figures that macro-cell PDP required larger CP length than micro-cell PDP because the macro-cell has a larger delay spread. It can be deduced from these simulations that different channels require different $\mathrm{CP}$ lengths corresponding to their MEDS; hence no single standard value of $\mathrm{CP}$ is optimal across all channel models.

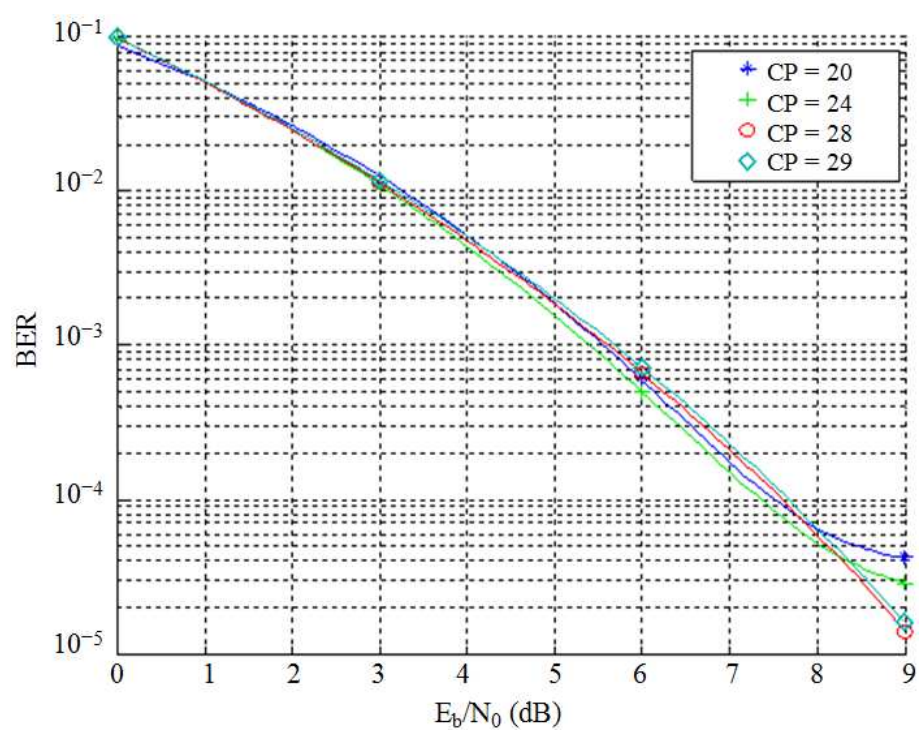

Fig. 10. BER for EVA PDP with different CP lengths

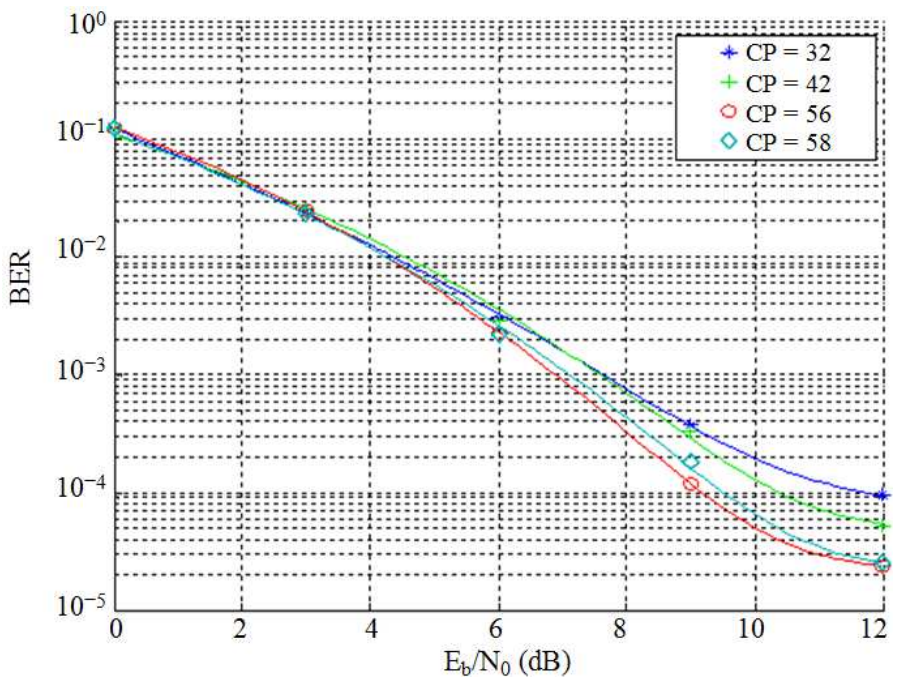

Fig. 11. BER for ETU PDP with different CP lengths 


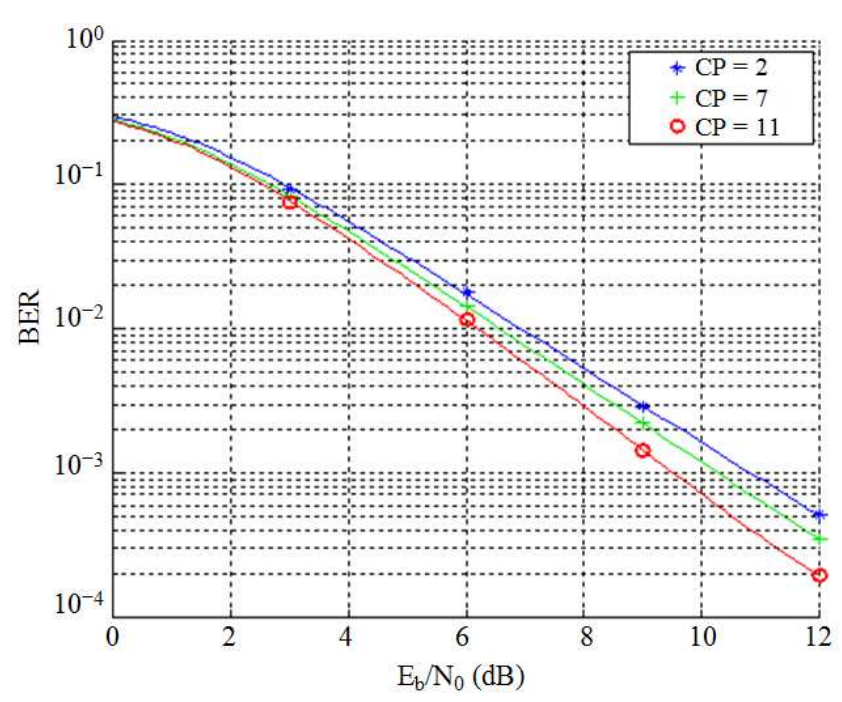

Fig. 12. BER for urban micro-cell PDP with different CP lengths

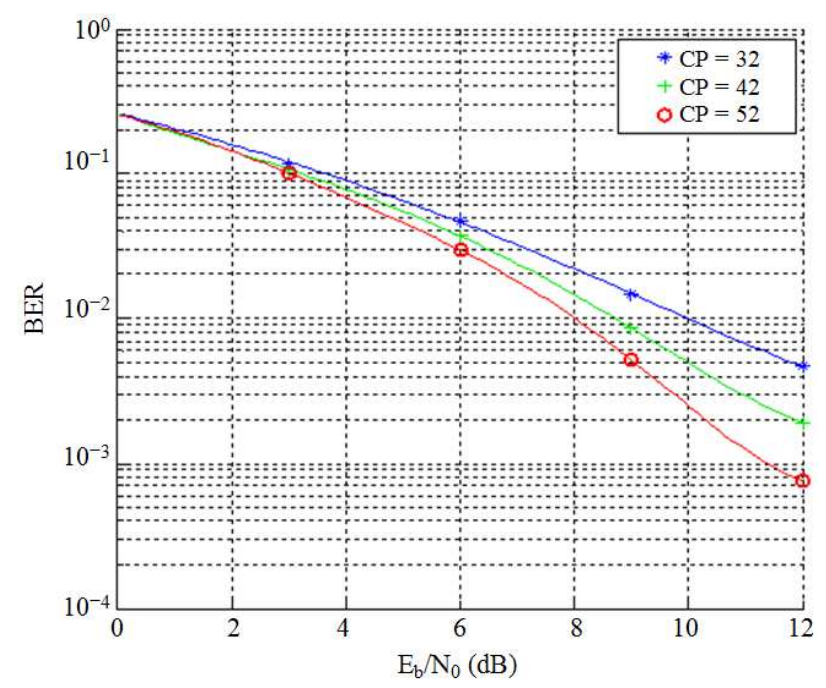

Fig. 13. BER for urban macro-cell PDP with different CP lengths

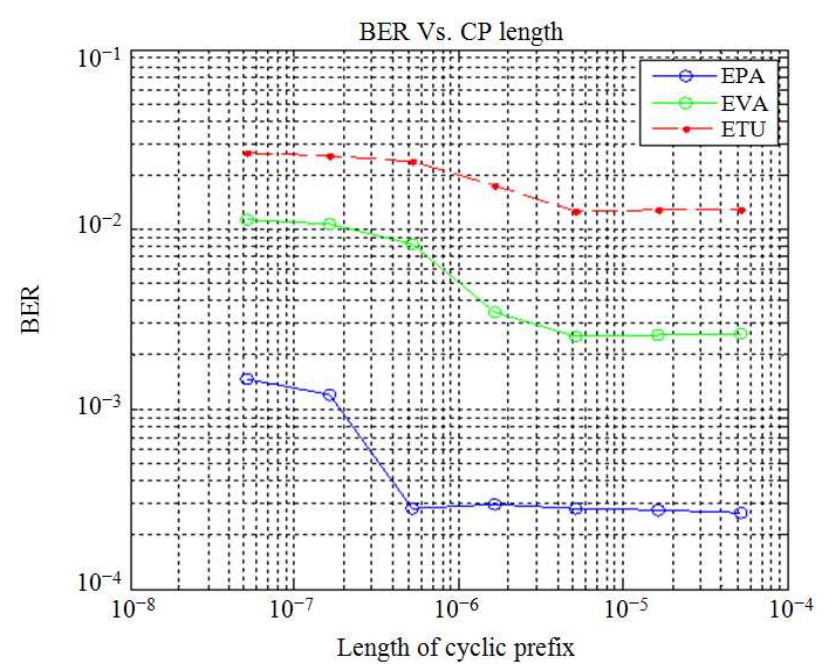

Fig. 14. BER Vs. CP lengths for EITU PDP 


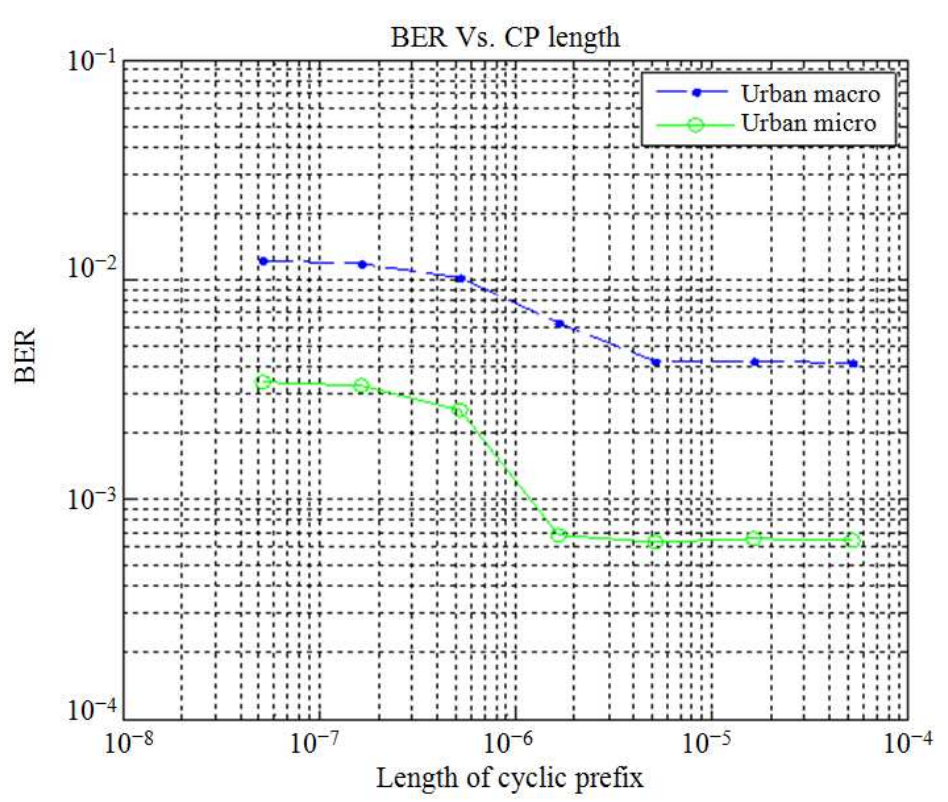

Fig. 15. BER Vs. CP lengths for 3GPP PDP

Table 8. OFDM data rate and SNR loss over ITU-B channel model

\begin{tabular}{llll}
\hline Channel type & Optimal CP length & Data rate $(\mathrm{Mbps})$ & Loss $(\mathrm{dB})$ \\
\hline EPA & $5(0.46 \mu \mathrm{s})$ & $12.188 \mathrm{Mbps}$ & $0.0218 \mathrm{~dB}$ \\
EVA & $29(2.81 \mu \mathrm{s})$ & $11.884 \mathrm{Mbps}$ & $0.131 \mathrm{~dB}$ \\
ETU & $56(5.6 \mu \mathrm{s})$ & $11.542 \mathrm{Mbps}$ & $0.258 \mathrm{~dB}$ \\
\hline
\end{tabular}

To summarize the analysis in previous section, Fig. 14 and 15 shows the effect of varying the CP length on the BER performance of the OFDM system over EITU and 3GPP channel models buttressing the optimal CP length required in terms of the BER Vs. CP lengths. From Fig. 14 and 15 we observe that increasing the CP length beyond the optimal length does not result in further improvement of the BER of the channel.

\section{Conclusion}

An adaptive OFDM system with variable CP length over different channel models with different PDPs was described in this study. It presented a method used to estimate the CP length based on the MEDS of the channel. According to this method, the $\mathrm{CP}$ length is chosen to improve the system performance and to optimize the system capacity by mitigating both ISI and ICI. The results proved that the variable CP length is useful for OFDM system operating on time dispersive channel. Furthermore, the optimal CP length is strongly dependant on Maximum Excess Delay Spread of the channel. It is found that the optimal CP minimizes the SNR degradation caused by ISI and maximizes the data rate of the system. These results also showed that increasing the CP length beyond the optimum length does not result in further improvement of the system performance.

Finally, it may be concluded that the $\mathrm{CP}$ length is an essential aspect of the OFDM system. When the CP selected optimally based on the channel parameters, the overall system performance is improved. This is accomplished through effectively mitigating the dominant problem (ISI and ICI) in the OFDM system.

\section{Acknowledgment}

Support of A. Al-Jzari by Higher Committee for Education Development in Iraq is acknowledgment.

\section{Author's Contributions}

A. Al-jzari: Participated in simulating and analyzing data and contributed to writing of the manuscript.

K. Ivica: Participated in analyzing and contributed to writing of the manuscript.

\section{Funding Information}

A portion of this work was funded through the Higher Committee for Education Development in Iraq.

\section{Ethics}

This work has not been published elsewhere. 


\section{Reference}

Das, S.S., F.H. Fitzek, E.D. Carvalho and R. Prasad, 2005. Variable guard interval orthogonal frequency division multiplexing in presence of carrier frequency offset. Proceedings of the IEEE Global Telecommunications Conference, Dec. 2-2, IEEE Xplore Press, St. Louis, MO., pp: 5-5. DOI: $10.1109 /$ GLOCOM.2005.1578296

Ghosh, S., 2011. Performance study of WiMAX for different QoS parameters with varying cyclic prefix. PhD Thesis, Jadavpur University Kolkata.

Hassan, K., T.A. Rahman, M.R. Kamarudin and F. Nor, 2011. The mathematical relationship between maximum access delay and the RMS delay spread. Proceedings of the 7th International Conference on Wireless and Mobile Communications, Jun. 19-24, Luxembourg City, Luxembourg, pp: 18-23.

Kaur, S. and G. Bharti, 2012. Orthogonal frequency division multiplexing in wireless communication systems: A review. Int. J. Adv. Res. Comput. Eng. Technol., 1: 125-129.

Li, J. and M. Kavehrad, 1999. Effects of time selective multipath fading on OFDM systems for broadband mobile applications. IEEE Commun. Lett., 3: 332-334. DOI: $10.1109 / 4234.809526$

Lim, C., Y. Chang, J. Cho, P. Joo and H. Lee, 2005. Novel OFDM transmission scheme to overcome caused by multipath delay longer than cyclic prefix. Proceedings of the IEEE 61st Vehicular Technology Conference, May 30-Jun. 1, IEEE Xplore Press, pp: 1763-1767. DOI: 10.1109/VETECS.2005.1543624

Marchetti, N., M.I. Rahman, S. Kumar and R. Prasad, 2009. OFDM: Principles and Challenges. In: New Directions in Wireless Communications Research, Tarokh, V. (Ed.), Springer, New York, ISBN-10: 1441906738, pp: 29-62.
Ness, R., J.P. Linnartz, L. Van der Perre and M. Engels, 2002. The OFDM Principle. In: Wireless OFDM Systems: How to Make Them Work? Engels, M. (Ed.), Springer, ISBN-10: 1402071167, pp: 33-51.

Nolan, K.E., 2005. Reconfigurable OFDM systems. PhD Thesis, Trinity College Dublin.

Payaswini, P. and D.H. Manjaiah, 2013. Performance analysis of link going down threshold for Media Independent Handovers. Proceedings of the International Conference on Emerging Trends in Communication, Control, Signal Processing and Computing Applications, Oct. 10-11, IEEE Xplore Press, Bangalore, pp: 1-5. DOI: $10.1109 / C 2 S P C A .2013 .6749353$

Prasad, R., 2004. OFDM for wireless communications systems. Artech House.

Shah, D.C., B.U. Rindhe and S.K. Narayankhedkar, 2010. Effects of cyclic prefix on OFDM system. Proceedings of the International Conference and Workshop on Emerging Trends in Technology, Feb. 26-27, ACM New York, pp: 420-424.

DOI: $10.1145 / 1741906.1741996$

Zhang, Y. and H. Liu, 2006. Impact of time-selective fading on the performance of quasi-orthogonal space-timecoded OFDM systems. IEEE Trans. Commun., 54: 251-260. DOI: 10.1109/TCOMM.2005.863773

Zhang, Z.Y. and L.F. Lai, 2004. A novel OFDM transmission scheme with length-adaptive cyclic prefix. J. Zhej. Univ. Sci., 5: 1336-1342.

DOI: $10.1007 / \mathrm{BF} 02937044$ 